\title{
La vitamine $D$, une nouvelle panacée ?
}

\section{Michèle GARABÉDIAN}

Hôpital Saint Vincent de Paul, 82

avenue Denfert-Rochereau, 75014, Paris

$<$ cnrs.svp@wanadoo.fr>

\begin{abstract}
Besides its well known anti-rachitic properties, vitamin $D$ has numerous in vitro and in vivo actions on the proliferation-differentiation of epidermal and cancer cells, as well as on the recruitment, differentiation and activities of the immune cells. Even though numerous association studies have suggested a possible link between a low vitamin D status and the risk of developing tumoral, auto-immune, neurologic or cardiovascular diseases, further data have proven to be more discordant and at best modest. In addition, the range of vitamin $D$ doses or status active on these extra-skeletal pathologies remains poorly defined, between insufficiency and overload. Looking forward to clearly needed randomized intervention studies, optimal vitamin D status and intakes remain best defined on the basis of the vitamin D indisputable effects on calcium homeostasis and skeletal mineralization.
\end{abstract}

Key words: vitamin D, osteoporosis, cancer, autoimmune diseases, 25-hydroxyvitamin D

forme active. La 1,25-(OH $)_{2} \mathrm{D}$ est alors considérée comme une hormone "calciotrope ", en raison de ses effets positifs à très faible concentration sur l'absorption intestinale du calcium et des phosphates, sur la libération du minéral osseux, et sur la minéralisation du squelette, mais aussi à cause de sa production dans les reins, à distance de ses sites d'action, et du contrôle étroit de cette production en fonction des besoins de l'organisme en calcium et phosphore. À partir de 1971 est mise en place toute une série d'études expérimentales, notamment en cultures cellulaires, qui vont préciser le mode d'action de la 1,25-(OH) 2 D sur les différentes cellules osseuses, l'intestin, le rein, les parathyroïdes, et dévoiler ses effets sur la différenciation de nombreux types cellulaires, tant osseux qu'extra-osseux, les fonctions immunitaires, la fertilité, les sécrétions hormonales, le système cardiovasculaire, le système nerveux et la fonction musculaire (Garabedian, 2000). Une meilleure compréhension du mode d'action intracellulaire de cette molécule, ainsi que l'identification de son récepteur, le VDR, et de ses interactions avec des régions régulatrices de multi- ples gènes, viennent encore étayer le concept d'une vitamine multifonction dont les actions dépassent largement le cadre de l'homéostasie calcique et de la minéralisation du squelette. En parallèle, sont synthétisés la 25-(OH)D, la 1,25$(\mathrm{OH})_{2} \mathrm{D}$ et plusieurs analogues actifs, dont la 1alpha $(\mathrm{OH}) \mathrm{D}_{3}$, et sont développés des dosages de ces métabolites qui permettent d'affiner la définition, la prévenion et les stratégies thérapeutiques des différentes formes de rachitisme/ostéomalacie et d'hypocalcémie, qu'elles soient nutritionnelles ou résistantes, héréditaires ou acquises. Le champ d'application de la vitamine $D$ et de ses dérivés reste cependant restreint à ces pathologies, le risque incontestable de surcharge calcique en limitant I'utilisation dans les pathologies non associées à un déficit en calcium ou en phosphore, telles que les pathologies tumorales, immunitaires, nerveuses, ou cardiovasculaires, par exemple.

Par ailleurs, la mesure des concentrations circulantes en 25-(OH)D est rapidement considérée comme étant le meilleur moyen d'évaluer le statut en vitamine $D$ d'un individu. Mais le développement

Pour citer cet article : Garabédian M. La vitamine D, une nouvelle panacée ? OCL 2011 ; 18(2) : 88-93. doi : 10.1684/ocl.2011.0373 
des outils informatiques et statistiques et la mise en place à partir des années 2000 d'études d'association de grande ampleur entre risque de développer diverses pathologies et apport/statut en vitamine $D$ confortent le concept de vitamine "multifonction" pour la vitamine $D$, viennent révolutionner la définition jusque-là consensuelle de déficience/insuffisance en vitamine $D$ et poussent à remplacer la notion de statut "normal " par celle de "statut souhaitable » en vitamine $D$.

Enfin, les besoins en vitamine D apparaissent de plus en plus comme dépendant de facteurs tels que l'âge, en plus des déterminants de l'apport exogène et endogène de vitamine $D$ que sont la pigmentation cutanée et les conditions de vie (latitude de résidence, exposition solaire, alimentation). Ces besoins sont les plus élevés pendant la croissance fœtale et post-natale, culminant en fin de grossesse, pendant les deux premières années de vie et à I'adolescence. Mais ils sont également élevés à l'autre extrême de la vie, alors que se développe une résistance à la vitamine $\mathrm{D}$, liée à une moindre production de $1,25-(\mathrm{OH})_{2} \mathrm{D}$, à une moindre expression de son récepteur VDR, et à une moindre capacité de l'intestin à absorber le calcium. Une étape supplémentaire est franchie avec l'identification, à partir de 1992, de polymorphismes sur le gène codant le récepteur VDR, puis de variants fréquents sur le gène codant CYP27B1, le cytochrome P450 de la 1-hydroxylase rénale. En effet, la mise en évidence d'associations entre génotype VDR et certains traits phénotypiques tels que le risque de fractures chez les sujets âgés (Fang et al., 2005), ou le gain de masse osseuse chez l'adolescente (Esterle et al., 2009), suggèrent l'existence de sous-groupes de populations génétiquement "résistants " ou " hypersensibles » à l'action de la vitamine D.

Ces révolutions dans notre appréhension des fonctions de la vitamine $D$ sont à l'origine d'un engouement notable pour la prise et la prescription de cette vitamine. Mais les données les plus récentes apportent quelques bémols à cet enthousiasme. Le présent article se propose de faire le point sur les résultats des études et méta-analyses les plus récentes, en se concentrant sur les rares études $d$ 'intervention contre placebo actuellement disponibles.

\section{La vitamine $D$, facteur reconnu de prévention et de traitement des défauts de minéralisation osseuse}

Le bénéfice apporté par la vitamine $D$ pour la prévention et le traitement du rachitisme chez l'enfant n'est plus à démontrer, comme en témoigne la réduction majeure de l'incidence du rachitisme, de 20 à 0,03\% aux ÉtatsUnis et de 15-26 \% à 0,06 \% en France, ayant suivi la mise en place de prophylaxies systématiques chez les nourrissons (Rizzoli et al., 2010). Les valeurs circulantes de $25-(\mathrm{OH}) \mathrm{D}$ atteintes pendant la première année de vie avec ces prophylaxies s'étagent entre 50 et $100 \mathrm{nmol} / \mathrm{L}^{1}$ (20 à $40 \mathrm{ng} / \mathrm{mL}$ ), en dépit de divergences quant aux apports conseillés dans ces deux pays : 25$37,5 \mu \mathrm{g} /$ jour $\quad(1000-1500 \mathrm{Ul} / \text { jour })^{2}$ en France et 5-25 $\mu \mathrm{g} /$ jour aux ÉtatsUnis (Rizzoli R et al., 2010 ; Harris, 2005 ; Wagner et Greer, 2008).

À l'adolescence, le rachitisme est beaucoup plus rare et limité à des populations cumulant plusieurs facteurs défavorables :

- forte pigmentation de la peau ;

- défaut d'exposition solaire ;

- non-consommation d'aliments sources de vitamine $D$ (poissons gras de mer et aliments enrichis essentiellement) ;

- déficit marqué d'apport calcique (moins de la moitié des apports nutritionnels conseillés, ANC);

- pathologies hépatodigestives ou rénales augmentant les pertes de vitamine D ; - pathologies à type de granulomatose augmentant le catabolisme de la vitamine D.

Mais une carence plus modérée dans ces populations à risque peut être plus fréquemment responsable d'un défaut d'acquisition de la masse osseuse, dont les conséquences à long terme mériteraient d'être précisées. Plusieurs études d'association et quelques études d'intervention sont disponibles. Elles suggèrent un retard à I'acquisition de la masse osseuse pour des valeurs circulantes de 25-(OH)D en dessous d'un seuil de $30-40 \mathrm{nmol} / \mathrm{L}$, et

\footnotetext{
${ }^{1}$ Les valeurs circulantes de $25-(\mathrm{OH}) \mathrm{D}$ sont habituellement exprimées en $\mathrm{mmol} / \mathrm{L}$ ou en $\mathrm{ng} / \mathrm{mL}(1 \mathrm{ng} / \mathrm{mL}=2,5 \mathrm{nmol} / \mathrm{L})$.

${ }^{2}$ les apports en vitamine $D_{2}$ ou $D_{3}$ sont exprimés en Unités Internationales (UI) ou en microgrammes $(1 \mu \mathrm{g}=40 \mathrm{UI})$.
}

un effet positif de la vitamine $D$ sur le gain de masse osseuse, qui font actuellement proposer un apport de doses quotidiennes de 5 à $15 \mu \mathrm{g}$ de vitamine $\mathrm{D}$ aux adolescents (Rizzoli et al., 2010 ; Wagner et Greer, 2008 ; Ross et al., 2011), ou de doses trimestrielles de 2,5 mg (Duhamel et al., 2000), cet apport étant systématique ou limité aux populations à risque et au moins en période hivernale.

À l'autre extrême de la vie, la carence en vitamine $D$ entraîne un défaut de minéralisation de la trame osseuse (ostéomalacie) mais aussi une hyperparathyroïdie qui accélère la perte osseuse liée au vieillissement (ostéoporose). Les résultats de nombreuses études d'intervention randomisées contre placebo montrent de façon concordante qu'une supplémentation en vitamine $D$ diminue la perte osseuse et réduit de 12-20\% le risque de fractures chez les personnes âgées de 65 ans et plus (Bishoff-Ferrari et al., 2009a ; Cranney et al., 2007), principalement chez celles placées en institution. Cet effet est associé à une correction de I'hyperparathyroïdie secondaire, et donc à une meilleure minéralisation osseuse, mais aussi à une augmentation de la force musculaire et à une réduction du nombre de chutes (Bischoff-Ferrari et al., 2009b). II requiert pour la plupart des auteurs un apport de vitamine D de 17-20 $\mu \mathrm{g} /$ jour correspondant à des valeurs circulantes de $25-(\mathrm{OH}) \mathrm{D}$ supérieures à $50-75 \mathrm{nmol} / \mathrm{L}$ (Ross et al., 2011 ; Bishoff-Ferrari et al., 2009a). II pourrait être plus marqué quand l'apport de vitamine D est associé à un supplément calcique de 500$1200 \mathrm{mg} /$ jour (Cranney et al., 2007), mais ceci $n^{\prime}$ a pas été retrouvé dans d'autres méta-analyses (Bishoff-Ferrari et al., 2009a).

En revanche, le lien entre santé osseuse et statut en vitamine D n'est encore clairement établi chez l'adulte que chez les personnes âgées (Seamans et Cashman, 2009), les études disponibles étant trop rares et parcellaires chezl'adulte jeune, et en particulier chez la femme avant la ménopause (Cranney et al., 2007).

\section{La vitamine $D$, facteur indispensable pour la prévention des hypocalcémies néonatales}

De par son action sur l'absorption intestinale du calcium et la libération 
du minéral osseux, la vitamine $D$ augmente la calcémie, et sa carence entraîne une hypocalcémie avec hyperparathyroïdie secondaire. Ces hypocalcémies ont une symptomatologie bruyante et parfois sévère en période néonatale, et l'apport de vitamine D chez la femme enceinte a un impact positif évident sur l'incidence des hypocalcémies néonatales chez la femme enceinte vue en période hivernale (Mallet et al., 1992 ; Zeghoud et al., 1997), avec une chute du nombre d'observations d'hypocalcémies néonatales symptomatiques de 7,7 à 2,4 pour 1000 naissances chez les femmes ayant reçu de la vitamine $D$ pendant leur grossesse (Mallet et al., 1992). Un apport systématique de vitamine $\mathrm{D}_{3}$ (2 à $2,5 \mathrm{mg}$ au $6^{\mathrm{e}}$ ou $7^{\mathrm{e}}$ mois de grossesse) est depuis ces études proposé aux femmes enceintes devant accoucher entre février et juin et à celles, quelle que soit la saison, qui ne s'exposent pas au soleil, ce traitement augmentant les valeurs circulantes de 25$(\mathrm{OH}) \mathrm{D}$ de $31 \pm 1$ à $50 \pm 2 \mathrm{mmol} / \mathrm{L} \mathrm{chez}$ la mère au moment de l'accouchement (Zeghoud et al., 1997).

\section{La vitamine $D$, facteur controversé de prévention des pathologies cancéreuses}

Les études expérimentales chez l'animal et in vitro ont montré sans ambiguiité que la 1,25-(OH) $)_{2} \mathrm{D}$ favorise la différenciation de la plupart de ses cellules cibles, via une inhibition ou une stimulation de l'expression de proto-oncogènes, tels que c-myc et c-fos, selon le type cellulaire et l'état quiescent ou non de la cellule. La 1,25$(\mathrm{OH})_{2} \mathrm{D}$ exerce également des effets antitumoraux marqués sur toute une série de cellules tumorales qui expriment le VDR, en bloquant ces cellules en phase $\mathrm{G} 1$, en activant la synthèse des protéines p21 et p27, en contrôlant l'expression et I'action de proto-oncogènes, en favorisant l'apoptose de certaines lignées tumorales via une inhibition de la production de la protéine $\mathrm{BCl}-2$, et en inhibant I'angiogenèse péritumorale et le développement de métastases (Garabedian, 2000). Ces effets ont été amplement confirmés in vivo chez I'animal, avec une réduction de la survenue et de la taille de tumeurs telles que mélanome, ostéosarcome, tumeurs du sein ou du côlon, et un allongement de 30 à $50 \%$ de l'espérance de vie d'animaux leucémiques ou porteurs de tumeurs (Garabedian, 2000). Cependant, I'application de ces résultats en clinique humaine reste limitée par I'hypercalcémie résultant des fortes doses de 1,25- $(\mathrm{OH}) 2 \mathrm{D}$ requises.

Peu après ces travaux, les premières études $d^{\prime}$ association font apparaître la possibilité d'un lien entre accroissement du risque de survenue, ou de récidive, $d^{\prime}$ un cancer du sein, de la prostate ou du côlon, et insuffisance des apports, ou du statut, en vitamine D (Garabedian, 2000 ; Garland et al., 1999). Ces travaux ont été à l'origine d'un foisonnement de nouvelles études d'association dont les résultats sont apparus plus contestables, inconstants, et au mieux modestes (Ross et al., 2011 ; Cranney et al., 2007 ; Chung et al., 2009). De plus, le lien de causalité entre déficience en vitamine $D$ et cancer reste non prouvé même quand il est suggéré, trop d'éventuels facteurs de confusion n'ayant pas été pris en compte, tels que pollution, autres nutriments associés aux aliments riches en vitamine D, activité physique associée à l'exposition solaire, ou encore l'obésité qui réduit les concentrations circulantes de 25-(OH)D. Enfin, le sens de la relation cause/effet n'est pas clairement établi, la possibilité que les sujets affaiblis par la maladie tumorale aient des apports alimentaires plus faibles et s'exposent moins au soleil n'ayant pas été suffisamment explorée (Ross et al., 2011 ; Cranney et al., 2007).

Pour lever ces incertitudes, des études d'intervention sont nécessaires. Mais seules quelques études sont actuellement disponibles et les résultats en sont décevants car ils ne montrent qu'un effet modeste et non significatif de I'apport de vitamine D sur le risque de cancer colorectal (Huncharek et al., 2009) et le risque de cancer du sein (Gissel et al., 2008 ; Chlebowski et al., 2008 ; Michels et al., 2007).

\section{La vitamine $D$, adjuvant anti-tuberculeux dont I'efficacité serait restreinte à certains sous-groupes de patients}

La 1,25-(OH $)_{2}$ D a une efficacité certaine in vitro sur la différenciation et les activités des macrophages, et l'existence d'un système autocrine/paracrine pour la vitamine $D$ est bien établie au sein des granulomes (Garabedian, 2000). Dans ce système, les macrophages activés (ou plus rarement les lymphocytes) produisent de la 1,25$(\mathrm{OH})_{2} \mathrm{D}$ jusqu'à des concentrations locales capables d'activer la différenciation des monocytes/macrophages et leur transformation en cellules géantes, de favoriser la mycobactéricidie, et de contrôler l'activité du granulome en modulant la production et la réponse à de nombreuses cytokines, dont I'interféron gamma, IL-1, et les PGE2 (Hewison, 2011). En cas de granulomatoses étendues, incluant tuberculose, lèpre et sarcoïdose, la 1,25$(\mathrm{OH})_{2} \mathrm{D}$ produite localement est de plus déversée dans le sang, avec pour conséquence une augmentation de l'absorption intestinale du calcium responsable d' hypercalcémie, mais aussi un épuisement des réserves en vitamine D qui pourrait en retour aggraver la maladie. Un premier essai d'intervention confirme le niveau bas des réserves en vitamine $D$ chez les patients tuberculeux $(22,8 \mathrm{nmol} / \mathrm{L})$, mais ne montre un effet bénéfique de la vitaminothérapie $D$, associée au traitement anti-tuberculeux, que dans un sousgroupe de population portant un génotype VDR particulier (Martineau et al., 2011). Cette vitaminothérapie D ne doit donc être prescrite qu'avec circonspection, et ceci d'autant plus qu'elle comporte un risque non négligeable, mais non encore exploré, $\mathrm{d}^{\prime}$ induire une hypercalcémie et des calcifications des tissus mous, en raison de la production locale de 1,25-(OH)2D par le granulome.

\section{La vitamine $D$, facteur possible de prévention de certaines maladies auto-immunes chez le nourrisson}

Comme pour le cancer, les effets immunosuppressseurs reconnus de la $1,25-(\mathrm{OH})_{2} \mathrm{D}$ in vitro et chez I'animal faisaient envisager un rôle possible de la vitamine $D$ pour retarder ou prévenir la survenue des maladies auto-immunes chez I'homme (Garabedian, 2000). Mais les études d'association se sont révélées sources de résultats discordants ou modestes (Ross et al., 2011 ; Cranney et al., 2007). L'enthousiasme de départ devant les premières publica- 
tions montrant un lien entre exposition solaire, ou statut vitaminique $D$, et sévérité de maladies auto-immunes telles que la sclérose multiple, ou de leur rechute, a fait place à des controverses et interrogations comparables à celles suscitées par l'association vitamine D/ risque de maladie tumorale (Smolders, 2011). Actuellement, en l'absence de données solides issues d'études d'intervention, il paraît donc prématuré de tenter de définir un statut vitaminique $D$ optimal et des besoins en vitamine $D$ sur la base d'un possible risque de développement de maladies autoimmunes (Ross et al., 2011 ; Cranney et al., 2007 ; Smolders, 2011). Cependant, les études concernant la survenue de diabète de type I chez l'enfant jeune semblent plus solides. Elles montrent en effet une association entre niveau des apports de vitamine D administrés pendant la première année de vie et risque de développer un diabète juvénile de type I (Harris, 2005). D'après leurs auteurs, un tel risque diminue si le nourrisson reçoit au moins $2000 \mathrm{UI} /$ jour de vitamine D (50 $\mathrm{gg} /$ jour) pendant la première année de vie, plutôt que 200 unités/ jour (5 $\mu \mathrm{g} /$ jour). En l'attente d'études testant l'éventuel impact de doses intermédiaires, il paraît donc prudent de maintenir les apports recommandés en France depuis plus de 40 ans, même s'ils sont supérieurs à ceux recommandés aux États-Unis.

\section{La vitamine $D$, données insuffisantes pour affirmer un effet préventif sur la survenue de pathologies cardiovasculaires et la mortalité}

Enfin, plusieurs méta-analyses relatives à l'effet de suppléments de vitamine D sur les fonctions cardiovasculaires et la mortalité, ont été récemment publiées. La vitamine $D$ pourrait réduire le risque cardiovasculaire en supprimant les processus d'inflammation, en inhibant la prolifération des cellules musculaires vasculaires lisses, et en réduisant la pression artérielle via une expression réduite du gène codant la rénine, tout en augmentant, à l'inverse, le risque de calcifications vasculaires (Manson, 2010). Les études Framington Offspring et Health Profesionnals Follow-up de cohortes d'hommes, comme l'étude NHANES III de cohortes mixtes (Melamed et al., 2008), montrent un lien inverse entre niveaux de $25-(\mathrm{OH}) \mathrm{D}$ et risque de maladie coronarienne et de pathologie cardiaque mortelle (Manson, 2010). Mais ici encore de nombreux facteurs de confusion n'ont pas été pris en compte (Manson, 2010), et, comme pour le cancer, I'Institut de médecine américain (IOM) considère que les données issues de ces études d'association ne permettent pas de déterminer un statut, ou des apports, de vitamine D permettant de prévenir la survenue de pathologies cardiovasculaires (Ross et al., 2011). Quelques études d'intervention sont cependant disponibles, même si leur objectif premier était de tester un bénéfice éventuel de la vitamine D sur l'ostéoporose et/ou les fractures, et non sur les risques de mortalité, de cancer ou de maladie cardiovasculaire. Ainsi, une analyse de 18 études randomisées incluant 57311 participants recevant ou non 300 à 2000 Ul/jour de vitamine D, suggère un modeste effet bénéfique sur le risque de mortalité (risque relatif 0,93, intervalle de confiance à $95 \%$ : 0,87$0,99)$, sans que des seuils d'apport de vitamine $D$ ni de statut en vitamine $D$ n'aient pu être déterminés (Autier et Gandini, 2007). Mais cet effet n'a pas été observé dans une autreétude randomisée incluant 36282 femmes après la ménopause, l'apport de $10 \mu \mathrm{g} / \mathrm{jour}$ de vitamine $D$ et de $1000 \mathrm{mg} / \mathrm{jour}$ de calcium pendant une durée moyenne de 7 ans n'ayant pas induit chez ces femmes de diminution significative du risque de maladie coronarienne, ni des risques de pathologies vasculocérébrale, cardiaque, ou tumorale mortelles (LaCroix et al., 2009). Un moyen de résoudre ces discordances est de mettre en place des études d'intervention randomisées contre placebo, de grandes ampleurs, et ayant la prévention des pathologies cardiaques et tumorales comme objectif principal, telles que l'étude VITAL actuellement en cours (Manson, 2010). Cependant, les apports quotidiens de vitamine D préconisés (50 $\mu \mathrm{g} /$ jour) dans cette étude prospective, ainsi que le niveau souhaité des valeurs circulantes de 25$(\mathrm{OH}) \mathrm{D}$ à atteindre (75-100 nmol/L), excèdent les propositions les plus récentes de I'Institut de Médecine (Ross et al., 2011), et l'étude d'éventuels effets indésirables devrait donc y être associée.

\section{Les effets indésirables de la vitamine D}

Si les résultats des études actuelles d'association ou d'intervention entre vitamine $D$ et pathologies extra-osseuses sont le plus souvent décevants. Les mêmes études, ou d'autres, font apparaître la possibilité inverse que l'administration chronique de doses supraphysiologiques de vitamine $D$ puisse avoir des effets indésirables. Certains effets indésirables de la vitamine D étaient connus de longue date en cas d'intoxication sévère, notamment l'augmentation de la charge calcique, avec hypercalcémie et hyperphosphatémie responsables de calcifications extraosseuses, et avec hypercalciurie responsable de lithiase urinaire, et d'insuffisance rénale. Mais d'autres effets, survenant à moyen ou long terme avec des apports plus faibles, commencent à être publiés au fur et à mesure que les prescriptions de vitamine $D$ se généralisent et que leur posologie augmente (Ross et al., 2011). Ainsi, une association est suggérée entre valeurs élevées de $25-(\mathrm{OH}) \mathrm{D}$ circulante et risque accru de cancer du pancréas (Stolzenberg-Solomon et al., 2010), de la prostate, du sein et de l'œsophage (Toner et al., 2010). De même, l'étude d'association NHANES suggère un accroissement du risque de mortalité, quelle qu'en soit la cause, en cas de valeurs circulantes de $25-(\mathrm{OH}) \mathrm{D}$ basses, mais aussi en cas de valeurs élevées, supérieures à $100 \mathrm{nmol} / \mathrm{L}$ ) (Melamed et al., 2008). Par ailleurs, non seulement le statut vitaminique $D$, mais aussi les apports calciques devraient être considérés. Quelques études suggèrent en effet un lien entre apports calciques et altérations du myocarde chez des femmes après la ménopause (Bolland et al., 2008), ainsi qu'entre apports vitamino-calciques et le volume de lésions cérébrales en imagerie IRM chez les personnes âgées (Payne et al., 2008).

\section{Au total, quel statut vitaminique $D$ serait optimal et quels apports conseiller?}

Ainsi, après l'efflorescence des publications des années 2000 tendant à faire de 
la vitamine $\mathrm{D}$ une panacée universelle, I'enthousiasme est retombé, comme I'ont été quelques années auparavant les espoirs relatifs aux bêta-carotènes, acide folique, sélénium, et vitamines $C$ et $E$, pour la prévention de lésions cancéreuses. Devant l'inconsistance, la modestie et les difficultés d'interprétation des résultats des études d'association entre, d'une part, statut (ou apports) en vitamine $D$ et, $d^{\prime}$ 'autre part, cancer, maladies auto-immunes, maladies cardiovasculaires, et pathologies neurologiques, la tendance présente est de tenter de définir un statut optimal en vitamine $D$ en se basant essentiellement sur ses effets osseux (Ross et al., 2011 ; Cranney et al., 2007 ; Chung et al., 2009).

L'Institut de médecine américain propose ainsi un seuil de $25-(\mathrm{OH}) \mathrm{D}$ circulant de $40 \mathrm{nmol} / \mathrm{L}$ pour couvrir les besoins de $50 \%$ des individus, un seuil de $50 \mathrm{nmol} /$ $\mathrm{L}$ pour couvrir ceux de $97,5 \%$ des individus et un seuil supérieur tolérable de $125 \mathrm{nmol} / \mathrm{L}$, des valeurs plus élevées n'ayant pas fait la preuve d'une amélioration de leur efficacité et pouvant faire courir un risque de surcharge à des sousgroupes de populations vulnérables (Ross et al., 2011). D'après les conclusions du même Institut, ces valeurs circulantes pourraient être atteintes par un apport quotidien de $15 \mu \mathrm{g} / \mathrm{jour}$ chez les sujets de 1 à 70 ans, ayant une exposition solaire nulle ou minimale, et de $20 \mu \mathrm{g} /$ jour pour les sujets plus âgés. Ces « RDA " (besoins moyens + 2 écartstypes) permettraient de couvrir les besoins de $97,5 \%$ de la population nord-américaine en bonne santé (Ross et al., 2011). La multiplication dans un proche avenir des études d'intervention orientées vers les effets extra-osseux de la vitamine $D$, mais aussi vers ses possibles effets indésirables, devrait permettre de préciser ces recommandations, et de mieux cerner les sous-groupes de populations rendus plus vulnérables par leur âge ou leur génotype aux effets de la déficience ou de la surcharge en vitamine D.

\section{RÉFÉRENCES}

Autier P, Gandini S. Vitamin D supplementation and total mortality: a meta-analysis of randomized controlled trials. Arch Intern Med 2007 ; 167 : 1730-7.

Bishoff-Ferrari HA, Willett WC, Wong JB, et al. Prevention of nonvertebral fractures with oral vitamin D and dose dependency: a metaanalysis of randomized controlled trials. Arch Intern Med 2009 ; 169 : 551-61.

Bischoff-Ferrari HA, Dawson-Hughes B, Staehelin $\mathrm{HB}$, et al. Fall prevention with supplemental and active forms of vitamin D: a meta-analysis of randomized controlled trials. BMJ 2009b; 339 : b3692 (doi : 10.1136/bmj.b3692).

Bolland MJ, Barber PA, Doughty RN, et al. Vascular events in healthy older women receiving calcium supplementation: randomised control trial. Brit Med J 2008 ; 336 : 262-6.

Chlebowski RT, Johnson KC, Kooperberg C, et al. ; Women's Health Initiative Investigators. Calcium plus vitamin D supplementation and the risk of breast cancer. I Natl Cancer Inst 2008 ; 100 : 1581-191.

Chung M, Balk EM, Brendel M, et al. Vitamin $D$ and calcium: a systematic review of health outcomes. Evid Rep technol Assess(Full Rep) $2009 ; 183: 1-420$.

Cranney A, Horsley T, O'Donnell S, et al. Effectiveness and safety of vitamin $D$ in relation to bone health. Evid Rep Technol Assess (Full Rep) 2007 : 1-235.

Duhamel JF, Zeghoud F, Sempe M, et al. Prophylaxie de la carence en vitamine D chez l'adolescent et le preadolescent. Etude interventionnelle multicentrique sur les effets biologiques d'un apport répété de 100000 UI de vitamine $D_{3}$. Arch Pediatr 2000 ; $7: 148-53$.

Esterle L, Jehan F, Sabatier JP, Garabedian M. Higher milk requirements for bone mineral accrual in adolescent girls bearing specific caucasian genotypes in the VDR promoter. J Bone Miner Res 2009 ; 24 : 1389-97.

Fang $\mathrm{Y}$, van Meurs JB, d'Alesio A, et al. Promoter and $3^{\prime}$-untranslated-region haplotypes in the vitamin D receptor gene predispose to osteoporotic fractures: The Rotterdam study. Am J Human Genet 2005 ; 77 : 807-23.

Garabedian M. La vitamine D : les nouvelles fonctions d'une ancienne vitamine. OCL $2000 ; 7$ : 271-75. [http://www.jle.com/fr/ revues/agro_biotech/ocl/e-docs/00/03/34/ ED/article.phtml]

Garland CF, Garland FC, Gorham ED. Calcium and vitamin $\mathrm{D}$. Their potential roles in colon and breast cancer prevention. Ann N Y Acad Sci 1999 ; 889 : 107-19.

Gissel T, Rejnmark L, Mosekilde L, Vestgaard P. Intake of vitamin $D$ and risk of breast cancer- a meta-analysis. J Steroid Biochem Mol Biol 2008 ; 111 : 195199.

Harris SS. Vitamin D in type 1 diabetes prevention. J Nutr 2005 ; 135 : 323-5.
Hernigou P. Histoire du rachitisme, de I'ostéomalacie et de la vitamine D. Rev Rhum $1995 ; 62$ : 273-82.

Hewison M. Antibacterial effects of vitamin D. Nat Rev Endocrinol 2011, Jan 25 (Epub ahead of print).

Huncharek M, Muscat J, Kupelnick B. Colorectal cancer risk and dietary intake of calcium, vitamin $D$, and dairy products: a meta-analysis of 26,335 cases from 60 observational studies. Nutr Cance 2009 ; $61: 47-69$.

LaCroix AZ, Kotchen J, Anderson G, et al. Calcium plus vitamin $D$ supplementation and mortality in postmenopausal women: The women's health Initiative calciumvitamin $D$ randomized controlled trial. J Gerontol A Biol Sci Med 2009 ; 64 : 559-67.

Mallet $\mathrm{E}$, Henocq A, de Menibus $\mathrm{CH}$. Effets de la supplémentation de la mère en vitamine $D$ sur l'incidence de l'hypocalcémie néonatale. In : Sciences FM, ed. Journées Parisiennes de Pédiatrie, 1992. Paris, 1992 : 67-69.

Manson JE Vitamin D and the heart: why we need large-scale clinical trials. Cleveland Clinic Journal of Medicine 2010 ; 77 : 903-910.

Martineau AR, Timms PM, Bothamley GH, et al. High-dose vitamin $\mathrm{D}(3)$ during intensive-phase antimicrobial treatment of pulmonary tuberculosis: a double-blind randomised controlled trial. Lancet $2011 ; 377$ : 242-50.

Melamed ML, Michos ED, Post W, Astor B. 25-hydroxyvitamin $D$ levels and the risk of mortality in the general populations. Arch Intern Med $2008 ; 168$ : 1629-37.

Michels KB, Mohllajee AP, Roset-Bahmanyar E, Beehler GP, Moysich KB. Diet and breast cancer : a review of the prospective observational studies. Cancer 2007 ; 109 : 2712-49.

Payne ME, Anderson J], Steffens DC. Calcium and vitamin $\mathrm{D}$ intakes may be positively associated with brain lesions in depressed and non depressed elders. Nutr Res 2008 ; 28 : 285-292.

Riche MA. Rapport du Jury International. Exposition Universelle de 1889 à Paris.

Rizzoli R, Bianchi ML, Garabedian M, McKay $\mathrm{H}$, Moreno L. Maximizing Bone mineral mass gain during growth for the prevention of fractures in the adolescents and the elderly. Bone 2010 ; 46 : 294-305.

Ross AC, Manson JE, Abrams SA, et al. The 2011 report on dietary reference intakes for calcium and vitamin D from the Institute of Medicine: What clinicians need to know. J Clin Endocrinol Metab 2011 ; 96 : 53-8.

Seamans KM, Cashman KD. Existing and potentially novel functional markers of vitamin D status: a systematic review. Am / Clin Nutr $2009 ; 89$ : 1997S-2008S. 
Smolders J. Vitamin D and multiple sclerosis : correlation, causality, and controversy. Autoimmune diseases 2011, Article ID 629538 (doi : 10.4061/2011/629538).

Stolzenberg-Solomon RZ, Jacobs EJ, Arslan $\mathrm{AA}$, et al. Circulating 25-hydroxyvitamin D and risk of pancreatic cancer: Cohort consor- tium vitamin D pooling project of rarer cancers. Am / Epidemiol 2010 ; 172 : 81-93.

Toner CD, Davis CD, Milner JA. The vitamin D and cancer conundrum: aiming at a moving target. J Am Diet Assoc 2010 ; 110 : 1492-500.

Wagner CL, Greer FR. Prevention of rickets and vitamin $D$ deficiency in infants, children, and adolescents. Pediatrics $2008 ; 122$ : 1142-52.

Zeghoud F, Vervel C, Guillozo H, WalrantDebray O, Boutignon H, Garabedian M. Subclinical vitamin $\mathrm{D}$ deficiency in neonates: definition and response to vitamin $D$ supplements. Am J Clin Nutr 1997; 65: 771-8. 